\title{
Enhancing chemogenomics with predictive
}

\section{pharmacology}

Tim James, Adam Sardar and Andrew Anighoro*

Evotec (UK) Ltd. 114 Innovation Drive, Milton Park, Abingdon, Oxfordshire OX14 4RZ,

UK

\section{Contents of SI:}

Table S1. Sum of annotations for scaffolds shown in Figure 2.

Page S2

Equation S1. Decomposition via Bayes' rule of the Positive Predictive Value. Page S3 


\begin{tabular}{|c|c|c|}
\hline Status & Annotation Count & Scaffold Count \\
\hline Phase I & 12,947 & 124 \\
\hline Phase II & 38,247 & 310 \\
\hline Phase III & 46,263 & 263 \\
\hline $\begin{array}{c}\text { Preclinical or earlier } \\
\text { (1 annotation) }\end{array}$ & 103,172 & 103,172 \\
\hline $\begin{array}{c}\text { Preclinical or earlier } \\
\text { (2-5 annotations) }\end{array}$ & 366,944 & 125,739 \\
\hline Phase IV & 515,409 & 1,023 \\
\hline $\begin{array}{c}\text { Preclinical or earlier } \\
(6+\text { annotations })\end{array}$ & $4,356,785$ & 125,238 \\
\hline
\end{tabular}

Table S1: Sum of annotations for scaffolds shown in Figure 2. All compound structures with at least one single-protein target annotation were extracted from ChEMBL25 and their Murcko ring system scaffolds were computed using RDKit version 2019.03.1 via a python interface. There are more annotations for chemotypes that have entered Phase IV than for the entire bottom two-thirds least annotated portion of ChEMBL. Further, the most annotated third of pre-clinical ChEMBL has less than ten times more data points despite containing over one hundred times more scaffolds. 


$$
\begin{aligned}
P\left(D^{+} \mid M^{+}\right) & =\frac{P\left(M^{+} \mid D^{+}\right) P\left(D^{+}\right)}{P\left(M^{+}\right)} \\
& =\frac{P\left(M^{+} \mid D^{+}\right)}{P\left(M^{+} \mid D^{+}\right) P\left(D^{+}\right)+P\left(M^{+} \mid D^{-}\right) P\left(D^{-}\right)} P\left(D^{+}\right) \\
& =\frac{P\left(M^{+} \mid D^{+}\right)}{P\left(M^{+} \mid D^{+}\right) P\left(D^{+}\right)+P\left(M^{+} \mid D^{-}\right)\left(1-P\left(D^{+}\right)\right)} P\left(D^{+}\right) \\
& =\frac{T P R \times P(\text { Positive Incidence Rate })}{T P R \times P(\text { Positive Incidence Rate })+F P R \times(1-P(\text { Positive Incidence Rate }))}
\end{aligned}
$$

Equation S1: Decomposition via Bayes' rule of the Positive Predictive Value into three constituent parts: true positive rate (TPR), false positive rate (FPR) and the overall positive incidence rate $\mathrm{P}(\mathrm{D}+)$ (also known as the 'Prevalence'). The notation $\mathrm{P}(\mathrm{D}+\mid \mathrm{M}+)$, short for $P($ Data is Positive/ Model predicts Positive), refers to the probability that a data point is in reality active given that a statistical model has predicted it as active. $\mathrm{P}(\mathrm{M}+\mid \mathrm{D}+)$ is the model accuracy on the set of positive cases; the TPR. In contrast, $\mathrm{P}(\mathrm{M}+\mid \mathrm{D}-)$ is the model error on the set of negative cases; the FPR. The full PPV expression is equivalent to the number of true positives over the number of positive predictions. 